\title{
Price vs Quantity in Duopoly Supergames with Close Substitutes ${ }^{1}$
}

\author{
Svend A lb1/2kx L uca Lambertini\# \\ $x$ Centre for Industrial E conomics \\ University of Copenhagen \\ Studiestr $r^{1} / 2$ de 6 \\ DK -1455 Copenhagen K, Denmark \\ \# Dipartimento di Scienze E conomiche \\ Universita degli Studi di Bologna \\ Strada Maggiore 45 \\ I-40125 B ologna, I taly \\ fax 39-51-6402664 \\ e-mail lamberti@spbo.unibo.it
}

J uly 9, 1998

${ }^{1} \mathrm{~A}$ cknowledgements. The paper was written while the second author was at the Institute of E conomics, University of Copenhagen. We thank the audience at a seminar in Copenhagen for useful comments. The usual disclaimer applies. 


\begin{abstract}
A bstract
We model the non-cooperative choice between quantity and price in order to stabilize collusion, through two meta-games where each ${ }^{-} \mathrm{rm}$ alternatively considers its payo ${ }^{\circledR}$ in the market supergame as directly related to its own or the rival's ability to collude. In the ${ }^{-}$rst setting, (i) if cartel pro ${ }^{-}$ts are evenly split, ${ }^{-}$rms collude in prices irrespective of the degree of di ßerentiation, so that initially a Prisoners' Dilemma is observed, while for very close substitutes the outcome is Pareto-e \pm cient; (ii) if $\mathrm{N}$ ash bargaining is adopted, price setting is dominant when substitutability is low, while no dominant strategy exists when substitutability is high, and the game has two asymmetric equilibria. In the second setting, the Nash equilibrium is unique and Pareto-e \pm cient for the most part of the substitutability range, while again two asymmetric equilibria obtain when products are very close substitutes.

J EL classi ${ }^{-}$cation: C72, D 43, L 13

K eywords: supergame, prisoners' dilemma

R unning head: Price vs Quantity in Supergames
\end{abstract}




\section{Introduction}

There exists a relatively small literature concerning the choice of the market variable in order to enhance the sustainability of collusion (Deneckere, 1983, 1984; Majerus, 1988; R othschild, 1992; A lb²/k and Lambertini, 1998). The conclusions reached in these papers are rather contradictory and suggest that cartel stability is highly sensitive to both the number of ${ }^{-} \mathrm{rms}$ and the functional form selected for market demand. One of these contributions is of particular interest here, that of Deneckere (1983) who 'nds that, along most of the substitutability range, a quantity-setting cartel is more easily sustained than a price-setting cartel; the opposite obviously holds when goods are complements. However, neither D eneckere nor others take into account the possibility that ${ }^{-} \mathrm{rms}$ may choose to collude in heterogeneous variables. This amounts to saying that, while indeed quantity behavior appears to minimize the probability of defection by either ${ }^{-} \mathrm{rm}$ from the cartel agreement in a wide parameter range, it is far from obvious that this is also su \pm cient to ensure that ${ }^{-}$rms will actually collude in output levels. Lambertini (1997) analyses this issue in a framework where the usual repeated market game leads to a meta-game, which is not necessarily observable, whose object is precisely the selection of the most e \pm cient way to play the market supergame. The model can be interpreted as an extended game in two stages a la Hamilton and Slutsky (1990). Lambertini proves that, as long as products are relatively weak substitutes, the choice of the market variable in supergames relocates the Prisoners' Dilemma backwards, from the one-shot market game to the one-shot meta-game where ${ }^{-}$rms choose the variable.

We extend Lambertini's (1997) analysis to the case of close substitutes. ${ }^{1}$ We consider two alternative settings. In the ${ }^{-} \mathrm{rst}$, each ${ }^{-} \mathrm{rm}$ considers its payo ${ }^{\circledR}$ in the meta-game as decreasing in its own critical discount factor; in the second, in its rival's critical discount factor. In both settings ${ }^{-} \mathrm{rms}$ can decide whether to split cartel pro ${ }^{-}$ts evenly or divide them according to a Nash bargaining solution. In the ${ }^{-}$rst meta-game, we establish that when products are fairly imperfect substitutes a Prisoners' Dilemma initially emerges, in that price-setting is a dominant strategy but symmetric quantity-setting would be Pareto-optimal. This holds irrespectively of the rule adopted to split cartel pro' ${ }^{-} \mathrm{ts}$; however, the adoption of a Nash bargaining rule makes it easier to solve the Prisoners' Dilemma through a meta-repeated game. Then, when products are almost identical, the equilibrium outcome of the meta-game is sensitive to the splitting rule. In case of equal split, the game has a unique and Pareto-e \pm cient $N$ ash equilibrium where ${ }^{-} \mathrm{rms}$ are price-setters. In case of Nash bargaining, we have a chicken game with two

\footnotetext{
${ }^{1}$ Lambertini (1997) only considers weak substitutes, so that deviation from the collusive path does not make the cheating ${ }^{-} \mathrm{rm}$ a monopolist (see Deneckere, 1983).
} 
asymmetric Nash equilibria. In the second meta-game, the game initially has a unique and Pareto-e \pm cient Nash equilibrium where both ${ }^{-}$rms set output levels, while when products are very similar we obtain another chicken game with asymmetric equilibria.

The remainder of the paper is structured as follows. In Section 2 the basic model is laid out. Section 3 de ${ }^{-}$nes the generic meta-game. Section 4 contains the critical discount factors characterizing each repeated market game. The two alternative meta-games are dealt with in Section 5 . Section 6 provides concluding comments.

\section{The basic model}

T wo ${ }^{-} \mathrm{rms}$, labelled $\mathrm{i}$ and $\mathrm{j}, \mathrm{o}$ \&er a single product each. The marginal cost of production is assumed to be constant and equal across ${ }^{-} \mathrm{rms}$ and, hence, normalized to zero. The inverse demand function faced by ${ }^{-} \mathrm{rm}$ i is

$$
p_{i}=1 ; q_{i}{ }^{\circ} q_{j} ;
$$

where ${ }^{\circ}$ denotes the degree of substitutability between the two products. As mentioned in the introduction, in ordpr to complement the analysis in Lambertini (1997), we restrict ourselves to ${ }^{\circ} 2[\overline{3} ; 1 ; 1]:^{2}$

The direct demand function faced by ${ }^{-} \mathrm{rm} i \mathrm{is}$

$$
q_{i}=\frac{1}{1+{ }^{\circ}} i \frac{1}{1 i^{\circ 2}} p_{i}+\frac{\circ}{1 i^{\circ 2}} p_{j}:
$$

When instead ${ }^{-} \mathrm{rm} i$ acts as a quantity-setter while ${ }^{-} \mathrm{rm} \mathrm{j}$ is a price-setter, their respective demand functions are

$$
\begin{gathered}
p_{i}=1 ; \quad q_{i}+{ }^{\circ}\left(p_{j}+{ }^{\circ} q_{i} i 1\right) ; \\
q_{j}=1 ; p_{j} i{ }^{\circ} q_{i}:
\end{gathered}
$$

In the absence of production costs, each ${ }^{-} \mathrm{rm}^{\prime} \mathrm{s}$ pro ${ }^{-} \mathrm{t}$ function corresponds to revenue, $1 / 4=p_{i} q_{i}$ :

\section{The meta-game}

The concept of extended game is due to Hamilton and Slutsky (1990; HS henceforth). They consider the extension, out of real time, of the basic duopoly game taking place in real time, in order to endogenise ' $r m s^{\prime}$ choices as to the timing of moves in the market. This yields a two-stage game, where the ${ }^{-}$rst stage concerns

\footnotetext{
${ }^{2}$ We only consider substitutes (and not complements), since the model is symmetric. See Singh and Vives (1984) and Deneckere (1983, 1984).
} 
the timing, and the second describes market subgames. ${ }^{3}$ Their approach can be adopted to investigate the choice of the market variable as well.

To this aim, consider ${ }^{-}$rst an extended or meta-game where ${ }^{-}$rms choose the market variable, knowing that the ensuing market competition takes the form of a one-shot game $\mathrm{G}^{1}$. We shall adopt here a symbology which largely replicates that in HS (1990, p. 32). De- ne $i^{1 ; 1}=(N ; \S ; i)$ the extended game. The subscript indicates that both the extension and the basic market game are one-

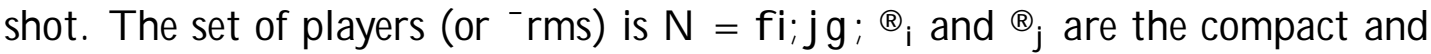
convex intervals of $R$ representing the actions available to $i$ and $j$ in the basic game. $\quad$ is the payo ${ }^{\circledR}$ function. Payo ${ }^{\circledR} S$ depend on the actions undertaken in the basic (market) game, according to the following functions, $1 / 4: \AA \_\AA$ ! $R$ and $1 / 4: \AA f \circledast$ ! $R$. The set of market variables from which ${ }^{-} r m s$ can choose is $V=f p$; qg. The set of strategies for player $i$ is $\S_{i}=f p$; $q g f C_{i}$, where $C_{i}$ is the

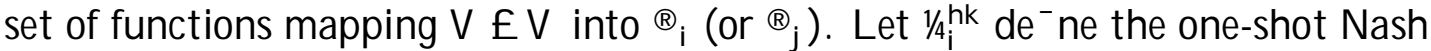
equilibrium pro ${ }^{-}$ts for ${ }^{-} \mathrm{rm} \mathrm{i}$, when she chooses market variable $\mathrm{h}$ and the rival chooses market variable k; with $h ; k 2 \mathrm{fp}$; qg: The reduced form of the meta-game can be described as in Matrix 1.

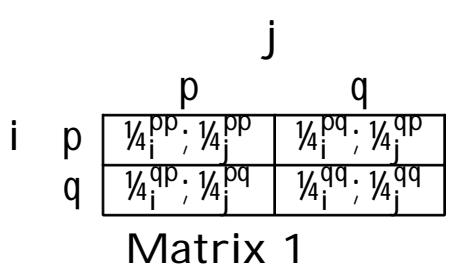

This is the game analysed by Singh and Vives (1984), using the demand functions introduced in the previous section. Since $1 / 4^{\mathrm{qp}}, 1 / \mathrm{pp}^{\mathrm{p}}$ and $1 / \mathrm{q}^{1 / \mathrm{q}}>1 / \mathrm{pq}^{\mathrm{pq}}$ for all ${ }^{\circ} 2(0 ; 1]$; they conclude that ${ }^{-}$rms play the symmetric Cournot equilibrium.

Consider now a meta-game $i^{1 ; 1}$, where the extension is again a one-shot choice over the set $\mathrm{V}=\mathrm{fp}$; qg; but market interaction takes place over an in ${ }^{-}$nite horizon (as in Lambertini, 1997), giving rise to a supergame $G^{1}$. Denote the individual discount factor of player i as $\sharp$ : In this case, ${ }^{-}$rms can collude in the relevant market variable(s). The relevant payo ${ }^{\circledR}$ function becomes $\phi$; and the individual payo ${ }^{\circledR}$ corresponds to the relevant critical level of the discount factor determined by repeated market interaction in each subgame, ț th: Accordingly, the reduced form of the extended or meta-game $i^{1 ; 1}$ is a $2 £ 2$ matrix analogous to $M$ atrix 1 , with $\pm_{4}^{\text {hk }}$ replacing $1 / 4^{\mathrm{k}}$ : G iven the symmetry of the model, one of the following situations may arise:

\footnotetext{
${ }^{3} \mathrm{HS}$ consider (i) an extended game with observable delay, where ${ }^{-} \mathrm{rms}$ declare the instant at which they will move, without announcing any particular action; and (ii) an extended game with action commitment, where ${ }^{-}$rms must commit to a speci ${ }^{-}$c price or quantity level. The meta-game we describe is conceptually similar to their game with observable delay.
} 
$2 i^{1 ; 1}$ is a coordination game, with equilibria $(p ; p)$ and $(q ; q)$;

$2 i^{1 ; 1}$ is a chicken game, with equilibria $(p ; q)$ and $(q ; p)$;

$2 i^{1 ; 1}$ has a unique equilibrium, either ( $\left.p ; p\right)$ or $(q ; q)$ : If so, two subcases are possible. Either the unique equilibrium is Pareto-e \pm cient, or not, i.e., the game is a Prisoners' Dilemma.

When $i^{1 ; 1}$ is a Prisoners' Dilemma, the question arises as to how ${ }^{-} \mathrm{rms}^{-}$can possibly solve it. If the choice of the market variable takes place in real time, then there would be no way out of such a Prisoners' Dilemma, in that, since $\mathrm{G}^{1}$ al ready takes place over an in ${ }^{-}$nite horizon, the repetition of $\mathrm{i}^{1 ; 1}$ over (real) time would require two contiguous and in ${ }^{-}$nitely long time intervals. On the contrary, if we adopt HS's view that the extension does not take place in chronological time, then we can envisage an extended game $i^{1 ; 1}$ where ${ }^{-}$rms interact in ${ }^{-}$nitely many times in order to select their respective market variables for the supergame in real time. This yields a critical discount factor ! : If individual meta-discount factors ! i are above ! , then 'rms solve the Prisoners' Dilemma. By adopting the simplifying assumption that $t=!_{i}$; i.e., ${ }^{-r m s}$ use the same discount factor in both supergames, we will identify conditions under which ${ }^{-} \mathrm{rms}$ may be able to solve both Prisoners' Dilemma, just one of them or neither.

\section{The critical discount factors}

Consider the market supergame $\mathrm{G}^{1}$ : To model collusion we adopt $\backslash$ grim" strategies (Friedman, 1971), where deviation in one period triggers a Nash punishment forever, as in Deneckere (1983), who obtained the following discount factors:

$$
\pm^{\mathrm{qq}}=\frac{\left({ }^{\circ}+2\right)^{2}}{{ }^{\circ} 2+8^{\circ}+8} ; \pm^{\mathrm{pp}}=\frac{\left(2 i^{\circ}\right)^{2}\left({ }^{\circ 2}+{ }^{\circ} i 1\right)}{\left(2 i^{\circ}\right)^{2}\left({ }^{\circ} 2+{ }^{\circ} i 1\right)+{ }^{\circ 4}} ;{ }^{\circ} 2^{\mathrm{hp}} \overline{3} ; 1 ; 1^{i} ;
$$

where superscript qq (pp) indicates that botp ${ }^{-} \mathrm{rms}$ set quantities (resp., prices). ${ }^{4}$ As $\pm^{q 9}< \pm^{\mathrm{pp}}$ for all ${ }^{\circ} 2 \overline{3}_{\overline{3}} 1 ; 0: 96155$; Deneckere (1983) concludes that Cournot behavior ensures greater stability of the cartel agreement than does B ertrand behavior, unless products are very close substitutes.

W hen ${ }^{-}$rms use heterogeneous variables the demand functions are (3) and (4). Straightforward computations yield the following Nash equilibrium pro ${ }^{-}$ts:

$$
1 / 4^{N}=\frac{\left({ }^{\circ} i 2\right)^{2}\left(1 ;{ }^{\circ 2}\right)}{\left(3^{\circ 2} i 4\right)^{2}} ; 1 / /^{\mathbb{N}}=\frac{\left({ }^{\circ} i 1\right)^{2}\left({ }^{\circ}+2\right)^{2}}{\left(3^{\circ} i_{4}\right)^{2}}
$$

\footnotetext{
${ }^{4}$ If ${ }^{\circ} 2\left(0 ;{ }^{p} \overline{3} ; 1\right)$; a di ßerent expression for $\AA^{p p}$ obtains. See Deneckere (1983, p. 41).
} 
where ${ }^{-} \mathrm{rm} \mathrm{i}$ is a quantity-setter, while ${ }^{-} \mathrm{rm} \mathrm{j}$ is a price-setter. The maximization of joint pro ${ }^{-}$ts requires $q_{i}^{\mathrm{M}}=1=\left(2\left(1+{ }^{\circ}\right)\right)$ and $p_{j}^{\mathrm{M}}=1=2:^{5} \mathrm{~T}$ his pair of strategies yields cartel pro ts : ${ }^{\mathrm{M}}=1=\left(2\left(1+{ }^{\circ}\right)\right)$ which we assume can be divided in two di ßerent ways. The ${ }^{-}$rst is that both ${ }^{-}$rms are entitled to half the cartel pro ${ }^{-}$ts, i.e., $1 / /^{M}=1 / \mathrm{p}^{\mathrm{M}}=1^{\mathrm{M}}=2$; the second consists in splitting evenly the additional pro ${ }^{-}$ts made available by collusion, in the light of the asymmetry between noncooperative pro $^{-}$ts:

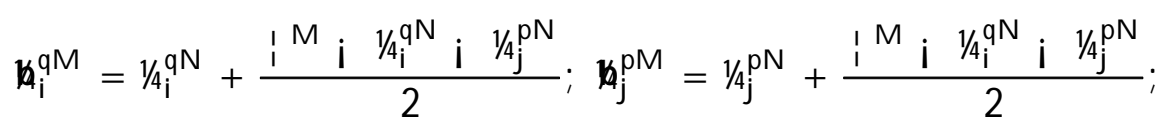

where obviously

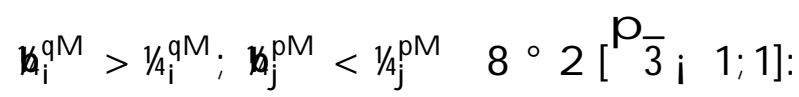

As to the deviation phase, the individually optimal deviation output (price) when the other ${ }^{-} r m$ sticks to the collusive price (output) corresponds to $\mathrm{q}_{\mathrm{D}}^{\mathrm{D}}=$ $1=\left(2^{\circ}\right)$ and $p_{j}^{D}=\left(2+{ }^{\circ}\right)=\left(4\left(1+{ }^{\circ}\right)\right)$. Notice that $q_{i}^{D}$ coincides with the monopoly output, given only one ${ }^{-} \mathrm{rm}$ on the market, since the deviation by the quantity setting ${ }^{-} \mathrm{rm}$ drives the price setting ${ }^{-} \mathrm{rm}$ completely out of the market. The corresponding deviation pro ${ }^{-}$ts are

$$
{ }_{1 / 4}^{1 / 4}=\frac{2^{\circ} i 1}{4^{\circ}} ; 1 / \% \mathrm{D}=\frac{\left({ }^{\circ}+2\right)^{2}}{16\left(1+{ }^{\circ}\right)^{2}}
$$

yielding the following pro ${ }^{-}$ts for the ${ }^{-} \mathrm{rm}$ being cheated:

$$
1 / \mathrm{p}^{\mathrm{Ch}}=0 ; 1 / 4 \mathrm{Ch}^{\mathrm{Ch}}=\frac{2+2^{\circ} \mathrm{i}^{\circ 2}}{8\left(^{\circ}+1\right)^{2}}
$$

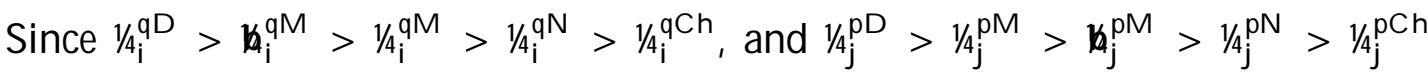
hold for the quantity-setter and the price-setter, respectively, the mixed situation where ' $r m s$ optimize in di ßerent variables reproduces the Prisoners' Dilemma, irrespectively of the rule adopted to split cartel pro ${ }^{-}$ts. As in the pure price or quantity games, a Pareto-improvement on the non-cooperative outcome can be reached in the repeated game over an in ${ }^{-}$nite horizon, if ${ }^{-} \mathrm{rms} i$ and $j$ 's discount factors are at least as high as the critical thresholds, de ned as:

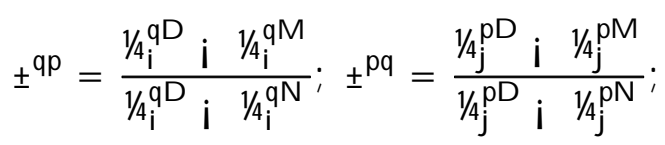

\footnotetext{
${ }^{5}$ Alternatively, ${ }^{-}$rms could resort to partial collusion. Moreover, they could adopt twostage optimal punishments (A breu, 1986, 1988; A breu, Pierce and Stacchetti, 1986). The view adopted here is meant to preserve the comparability with most of the existing literature.
} 
where superscript $\mathrm{qp}(\mathrm{pq})$ indicates that the discount factor is computed for the quantity-setter (price-setter), and $1 / 4^{\mathrm{M}}=1 / \mathrm{p}^{\mathrm{M}}=1^{\mathrm{M}}=2$ : A Iternatively, if rule (7) is adopted,

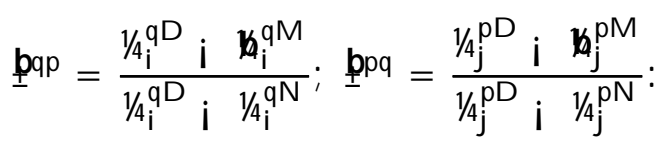

After some simple albeit tedious calculations, one - $n d s$ that the individual discount factors must satisfy the following inequalities:

$$
\begin{aligned}
& \pm . \quad \pm^{\mathrm{qp} p}=\frac{\left(3^{\circ 2} i 4\right)^{2}\left({ }^{\circ 2}+{ }^{\circ} i 1\right)}{\left(4^{\circ 7}+6^{\circ 6}+5^{\circ 5} i 29^{\circ 4} i 24^{\circ}+40^{\circ 2}+16^{\circ} i 16\right)}
\end{aligned}
$$

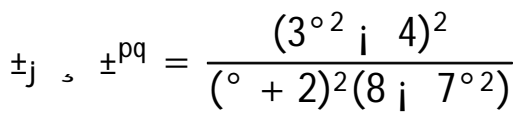

if cartel pro ${ }^{-}$ts are evenly split between the two ${ }^{-}{ }^{-} \mathrm{ms}$, or

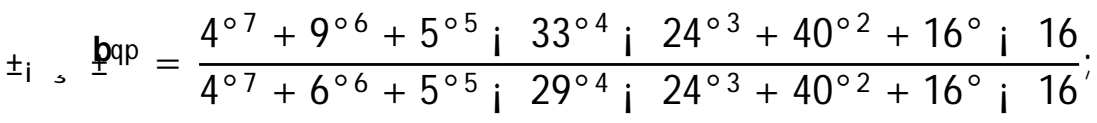

$$
\begin{aligned}
& \text { †, } \underline{\underline{p q}}=\frac{16+16^{\circ} \mathrm{i} 8^{\circ 2} \mathrm{i} 16^{\circ 3} \mathrm{i} 7^{\circ 4}}{\left({ }^{\circ}+2\right)^{2}\left(8 \mathrm{i} 7^{\circ 2}\right)}
\end{aligned}
$$

if cartel pro ts are split according to $(\mathrm{i} 7)$. These two pairs of critical discount factors can coexist when ${ }^{\circ} 2 \overline{3}_{i} 1 ; 1$ :

\section{The upstream stage}

We are now in a position to describe the one-shot meta-game $i^{1 ; 1}$ : Singh and Vives (1984) consider $i^{1 ; 1}$; establishing that if ${ }^{-} r m s$ selling substitute goods in a one-shot game can choose between setting price or quantity, they end up with a Cournot-Nash outcome, since setting output is a strictly dominant strategy. Lambertini (1997) addresses the same issue in a supergame, describing $i^{1 ; 1}$ over the range ${ }^{\circ} 2 \quad 0_{i} \overline{3}_{i} 1$. As mentioned in the introduction we extend his analysis in two ways; - rst, we look at close substitutes ( ${ }^{\circ} 2 \mathrm{hp}_{\overline{3}} ; 1 ; 1$ ); second, we analyse two meta-games. In Subsections 5.1 and 5.2 we assume, as in Lambertini (1997), that the payo ${ }^{\circledR}$ of the individual player is negatively related to the value of his own critical discount factor in the resulting repeated market game. In Subsection 5.3, we describe an alternative situation where each player knows his own discount factor, but not the rival's, and we argue that the payo ${ }^{\circledR}$ in the meta-game is then negatively related to the value of the rival's critical discount factor. 


\subsection{The supergame with equal split}

In this section we explore the case when the colluding ${ }^{-} \mathrm{rms}$ split the monopoly pro' ts equally.

Proposition 1 When the colluding ${ }^{-} \mathrm{rms}$ split thepmonopoly pro' ts equally, pricesetting is a strictly dominant strategy for all ${ }^{\circ} 2, \overline{3} ; 1 ; 1$ and weakly dominant for perfect substitutes. For ${ }^{\circ} 2{ }^{h} \overline{3}_{i} 1 ; 0: 961551$ the game $i^{1 ; 1}$ is a Prisoners' Dilemma while for ${ }^{\circ} 2[0: 961551 ; 1]$ the outcome is Pareto-e \pm cient.

Proof. The normal form of the upstream stage of the game is described by Matrix 2:

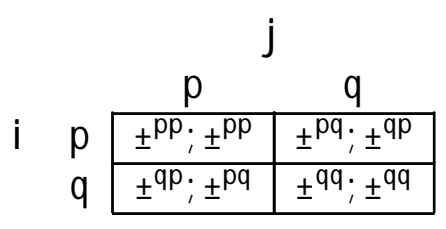

M atrix 2

It is easy to check that for all ${ }^{\circ} 2^{h p} \overline{3} ; 1 ; 1^{i}$

$$
\pm^{\mathrm{qp}}, \pm^{\mathrm{pp}} \text { and } \pm^{\mathrm{qq}}> \pm^{\mathrm{pq}}
$$

Hence, the game yields a unique equilibrium with both ${ }^{-} \mathrm{rms}$ setting prices. As noted by Deneckere (1983), $\pm^{q q}> \pm^{p p}$ for ${ }^{\circ} 2(0: 961551 ; 1]$ which proves the second part of Proposition 1.

\subsection{The supergame with $\mathrm{N}$ ash bargaining}

Here we consider the case where the ${ }^{-} \mathrm{rms}$ split the monopoly pro $^{-}$ts according to the rule (7).

Proposition 2 For all ${ }^{\circ} 2^{h p} \overline{3}_{i} 1 ; 0: 873108$ ' price-setting is a strictly dominant strategy, and the game $i^{1 ; 1}$ is a Prisoners' Dilemma. For all ${ }^{\circ} 2[0: 873108 ; 1]$ we have a chicken game with two asymmetric equilibria, $(p ; q)$ and $(q ; p)$.

Proof. In this case the normal form is as in Matrix 3: 


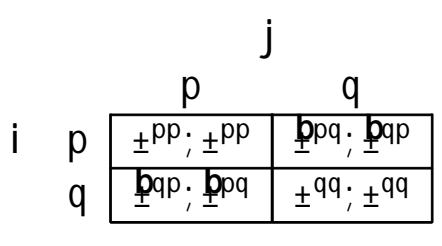

Matrix 3

One can check that

$$
\begin{aligned}
& \underline{4}^{\text {qp }}> \pm^{\text {pp }} \text { for all }{ }^{\circ} 2^{h p} \overline{3} ; 1 ; 0: 873108^{\prime}
\end{aligned}
$$

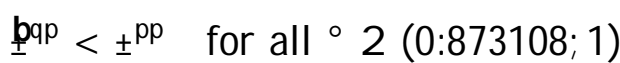

$$
\begin{aligned}
& \pm^{q q}>\text { pq for all }{ }^{\circ} 2^{h p} \overline{3} ; 1 ; 1
\end{aligned}
$$

which proves Proposition 6 .

For the range ${ }^{\circ} 2 \bar{P}_{i} 1 ; 0: 873108$; the results are largely analogous to those in Lambertini (1997). Thus, if ${ }^{\circ} 2{ }^{h p} \overline{3}$ i $1 ; 0: 873108$, a Prisoners' Dilemma arises independently of the rule adopted to split cartel pro ${ }^{-}$ts. Since - rms are playing a repeated game on the market, where they are trying to collude in a sustainable way, one can imagine that, in order to achieve the Pareto-superior outcome associated with collusion in quantities, - rms must repeat as well the interaction in the upstream stage in " nitely many times, building thus a metarepeated game $i^{1 ; 1}$ whose payo ${ }^{1} S$ are the critical discount factors displayed in matrices 1 and 2 . We then have

Proposition 3 When ${ }^{\circ} 2^{h p} \overline{3}$ i $1 ; 0: 873108$; ' rms can more easily obtain the Pareto-optimal outcome through a meta-repeated game if they adopt the Nash bargaining rule.

Proof. In Matrix 1 (resp., 2), the collusive payo ${ }^{\circledR}$ corresponds to \pm 99 , the noncooperative one to $\pm^{p p}$; the deviation one to $\pm^{\mathrm{pq}}$ (resp., ${ }^{\mathrm{ppq}}$ ), while - nally the

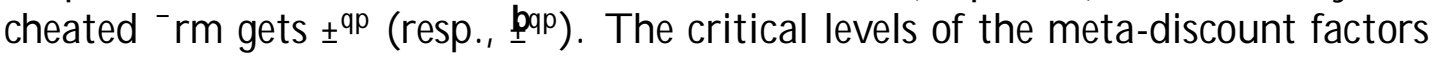
associated with the meta-repeated game are:

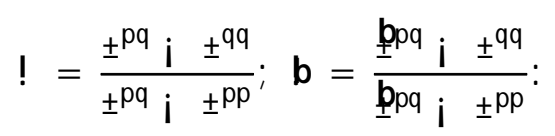

For collusion to be sustainable in the meta-repeated game, the individual meta-discount factors of players (which coincide in the meta-game) must satisfy either the following inequality: 


$$
!_{i}>!=\frac{16^{\circ}\left(1+{ }^{\circ}\right)^{2}\left(2^{\circ 4} i 3^{\circ 3} i^{\circ 2}+8^{\circ} i 4\right)\left({ }^{\circ 3}+6^{\circ 2} i 8\right)}{\left(8+8^{\circ}+{ }^{\circ} 2\right)\left(25^{\circ 8} i 20^{\circ} i 128^{\circ 6}+80^{\circ 5}+260^{\circ} i_{64^{\circ 3}} i 224^{\circ 2}+64\right)}
$$

or the alternative

$$
!_{\mathrm{i}}>\mathrm{b}=\frac{16^{\circ 3}\left(1+{ }^{\circ}\right)^{2}\left(2^{\circ 4} \mathrm{i}^{\circ 3} \mathrm{i}^{\circ 2}+8^{\circ} \mathrm{i} 4\right)}{7^{\circ} 10+60^{\circ} 9+120^{\circ} 8+336^{\circ}+508^{\circ}{ }^{\circ} 784^{\circ}{ }^{\mathrm{i}} 1792^{\circ}+448^{\circ}+1728^{\circ} \mathrm{i}^{5} 512^{\prime}}
$$

where ! 2 [0:80616; 0:872446) and b 2 [0:33641;0:653838); for ${ }^{\circ} 2^{\mathrm{hp}} \overline{3}$ i $1 ; 0: 87,3108$.

The di ßerence between ! and $b$ is decreasing in ${ }^{\circ}$, for all ${ }^{\circ} 2^{h p} \overline{3} ; 1 ; 0: 873108$; so rule (7) ensures higher stability, in that it yields a threshold which is lower than that obtained when cartel pro ${ }^{-}$ts are evenly distributed across ${ }^{-} \mathrm{rms}$.

As to the remainder of the parameter range, when ${ }^{\circ} 2[0: 873108 ; 1)$; the following holds. In the supergame with equal split, we know from Proposition 1 that the equilibrium ( $p ; p)$ is Pareto-e \pm cient for ${ }^{\circ} 2[0: 961551 ; 1]$. If the Nash bargaining rule is adopted, Proposition 2 establishes that both $(p ; q)$ and $(q ; p)$ are equilibria of the upstream stage. As a consequence, we can state

Proposition 4 For ${ }^{\circ} 2$ [0:873108; 0:961551); ' rms can attempt using a metarepeated game to obtain a Pareto-optimal outcome only in the game with equal split. For ${ }^{\circ} 2[0: 961551 ; 1)$; the Pareto-optimal outcome can be obtained in the game with equal split without resorting to the meta-repeated game.

Suppose ${ }^{-}$rms use the same individual discount factor both in the repeated market game and in the meta-repeated game. Call this discount factor $1 / 2 T$ hen, in order for the meta-game to solve the Prisoners' Dilemma, $1 / 2$ has to ful ${ }^{-} \mid$two conditions: it has to be greater than the critical level of the discount factor associated with the Cournot repeated game, and, at the same time, be greater than the critical threshold of the discount factor yielded by the meta-game. Since ! $>b$ for all ${ }^{\circ} 2 \overline{3}_{\mathrm{i}} 1 ; 0: 873108$; the necessary and su \pm cient condition in that range for the meta-game solving the Prisoner's Dilemma is $1 / 2$, maxf $\pm 9 q ;$ bg: For - 2 [0:873108; 0:961551) $r$ is not relevant and the condition is $1 / 2$, maxf $\pm 9 q ;$ ! $g$. Hence, we can state

Proposition $5 \mathrm{If}^{-} \mathrm{rms}$ use the same individual discount factorih the market and in the meta-repeated game, the binding constraint is \pm 99 for ${ }^{\circ} 2 \overline{3}_{\mathrm{i}} 1 ; 0: 835837$; b for ${ }^{\circ} 2$ (0:835837; 0:873108); while it is ! for ${ }^{\circ} 2$ (0:873108; 0:961551).

P roof. It can be easily checked that

$$
\text { ! }> \pm \pm^{q q}>b \quad 8^{\circ} 2^{h p} \overline{3} \text { i } 1 ; 0: 835837
$$




$$
\text { ! > b > } \pm^{99} \quad 8^{\circ} 2(0: 835837 ; 0: 873108) \text {; }
$$

Consider ${ }^{-}{ }^{-r s t}{ }^{\circ} 2{ }^{h p} \overline{3}_{i} 1 ; 0: 835837:$ In this range, if ${ }^{-} r m s$ can collude in the repeated Cournot game, they can also escape the Prisoners' Dilemma since $\pm 99>$ $\mathrm{b}$ : When ${ }^{\circ} 2(0: 835837 ; 0: 873108)$; the binding constraint is represented by the critical discount factor associated with the meta-game. Hence, ${ }^{-r m s}$ may be able to collude in the repeated Cournot game but not to escape the P risoners' Dilemma since there is the possibility that $b>1 / 2> \pm 9$ : For ${ }^{\circ} 2(0: 873108 ; 0: 961551), r$ is not relevant, and ! becomes the binding constraint since it is always greater than $\pm^{9 \%}$ in that range.

\subsection{A n alternative meta-game}

In the meta-game we have discussed so far, we assumed that the payo ${ }^{\circledR}$ of the individual player was negatively related to the value of his own critical discount factor in the resulting repeated market game. One could, however, imagine a situation where each player knows his own discount factor, but not the rival's. Suppose too that a player knows that he himself is able to collude and wants to maximize the possibility that the other player is also able to do so. In that case, the payo $\AA$ in the meta-game is negatively related to the value of the rival's critical discount factor, i.e., in matrices 1 and 2 player i 's payo ${ }^{\circledR S}$ are those in the second row, and vice versa. In this game, the relevant comparisons are $\pm^{\mathrm{pp}}$ with

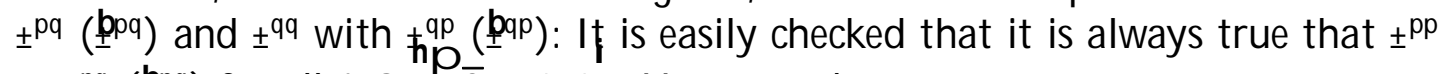

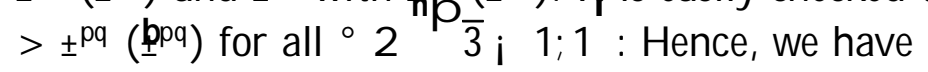

Proposition 6 The strategy pair ( $p ; p)$ can never be an equilibrium. If $\pm^{\mathrm{qp}}$ ( \pm qq; the only equilibrium is (q; q): If the opposite holds, we have a chicken game with two asymmetric equilibria.

Proof. To check this, we consider the two splitting rules ih turn.

In the meta-game with equal split, $\pm^{q p}> \pm \pm^{99}$ for all ${ }^{\circ} 2 \overline{3}_{1} 1 ; 0: 992028$ : In that range, ${ }^{-}$rms collude a la Cournot. Hence, for ${ }^{\circ} 2$ (0:961551; 0:992028]; this gives rise to another Prisoners' Dilemma, in that symmetric price behavior would yield a Pareto-superior outcome. ${ }^{6}$ For ${ }^{\circ} 2(0: 992028 ; 1]$; there is no dominant strategy and the repeated market game will be asymmetric with one ${ }^{-} \mathrm{rm}$ being

a quantity-setter and the other a price-setter.
With Nash bargaining, hap $> \pm \pm^{+99}$ for all ${ }^{\circ} \mathrm{hp}_{\overline{3}} ; \quad 1 ; 0: 936003$ ' and ' rms collude a la Cournot. In the remainder of the admissible range, we have again a chicken game with the resulting asymmetric repeated market interaction.

\footnotetext{
${ }^{6}$ The ' $r m s$ could then resort to a meta-repeated game to try to resolve this Prisoners' Dilemma. For sake of brevity we will not analyze this meta-repeated game.
} 


\section{Conclusions}

We have investigated the choice of the strategic variable in order to stabilize collusion in repeated duopoly games with close substitutes. To this aim, we have considered two alternative settings. In the ${ }^{-}$rst, each ${ }^{-}$rm perceives its payo ${ }^{\circledR}$ in the upstream stage as increasing in its own ability to stick to the collusive path. In the second, assuming it knows to be patient enough to stick to the collusive path independently of the variable selected, it considers its payo ${ }^{\circledR}$ as directly related to the rival's ability to collude. As a result, the ${ }^{-} \mathrm{rm}$ chooses the market variable that minimizes the rival's likelihood of deviating.

In the ${ }^{-}$rst setting, we established that, when products are still fairly imperfect substitutes, a Prisoners' Dilemma initially emerges in choosing the market variable irrespectively of the rule adopted to split cartel $\mathrm{pro}^{-} \mathrm{ts}$, although the adoption of a Nash bargaining rule yields a weaker requirement in view of escaping the P risoners' Dilemma through a meta-repeated game. When products are almost perfect substitutes, the equilibrium of the meta-game depends on the splitting rule. If cartel pro ts are equally split, the game has a unique and Pareto-e \pm cient Nash equilibrium where ${ }^{-}$rms are price-setters. In case of Nash bargaining, a chicken game obtains, with two asymmetric Nash equilibria. In the second setting, if substitutability is relatively low the game has a unique and $\mathrm{P}$ areto-e \pm cient $\mathrm{N}$ ash equilibrium where both ${ }^{-} \mathrm{rms}$ set output levels, while if substitutability is very high we obtain another chicken game. 


\section{R eferences}

[1] Alb $1 / 2 k$, S. and L. Lambertini (1998), Collusion in Di ßerentiated Duopolies Revisited, Economics Letters, forthcoming.

[2] A breu, D. (1986), Extremal Equilibria of Oligopolistic Supergames, J ournal of E conomic Theory, 39, 191-225.

[3] Abreu, D. (1988), On the Theory of In ${ }^{-}$nitely Repeated Games with Discounting, E conometrica, 56, 383-96.

[4] Abreu, D., D. Pearce and E. Stacchetti (1986), Optimal Cartel Equilibria with Imperfect M onitoring, J ournal of E conomic Theory, 39, 251-69.

[5] Deneckere, R. (1983), Duopoly Supergames with Product Di ßerentiation, E conomics Letters, 11, 37-42.

[6] Deneckere, R. (1984), Corrigenda, E conomics Letters, 15, 385-387.

[7] Friedman, J.W. (1971), A Non-Cooperative Equilibrium for Supergames, Review of Economic Studies, 28, 1-12.

[8] Hamilton, J . and S. Slutsky (1990), Endogenous Timing in Duopoly Games: Stackelberg or Cournot Equilibria, Games and Economic Behavior, 2, 29-47.

[9] Lambertini, L. (1997), Prisoners' Dilemma in Duopoly (Super)Games, J ournal of Economic Theory, 77, 181-91.

[10] Majerus, D. (1988), Price vs Quantity Competition in Oligopoly Supergames, Economic Letters, 27, 293-297.

[11] Rothschild, R. (1992), On the Sustainability of Collusion in Di Rerentiated Duopolies, Economic Letters, 40, 33-37.

[12] Singh, N. and X. Vives (1984), Price and Quantity Competition in a Di ßerentiated Duopoly, RAND J ournal of Economics, 15, 546-554. 